\title{
BMJ Open Cost-utility analysis of antimicrobial stewardship programme at a tertiary teaching hospital in Ethiopia
}

Gebremedhin Beedemariam Gebretekle (1) ,1,2,3 Damen Haile Mariam, ${ }^{4}$
Stephen Mac, ${ }^{2,3}$ Workeabeba Abebe ${ }^{5}$ Tinsae Alemayehu,, 6
Wondwossen Amogne Degu, ${ }^{8}$ Michael Libman, ${ }^{9}$ Cedric P Yansouni, ${ }^{9,10}$
Teferi Gedif Fenta, ${ }^{1}$ Makeda Semret, ${ }^{9}$ Beate Sander ${ }^{2,3}$
To cite: Gebretekle GB, Mariam DH, Mac S, et al. Costutility analysis of antimicrobial stewardship programme at a tertiary teaching hospital in Ethiopia. BMJ Open 2021;11:e047515. doi:10.1136/ bmjopen-2020-047515

- Prepublication history and additional supplemental material for this paper are available online. To view these files, please visit the journal online (http://dx.doi.org/10.1136/ bmjopen-2020-047515)

MS and BS are joint senior authors.

Received 01 December 2020 Accepted 17 November 2021

Check for updates

(C) Author(s) (or their employer(s)) 2021. Re-use permitted under CC BY-NC. No commercial re-use. See rights and permissions. Published by BMJ.

For numbered affiliations see end of article.

\section{Correspondence to}

Dr Gebremedhin Beedemariam Gebretekle;

gebremedhin.beedemariam@ aau.edu.et

\section{ABSTRACT}

Objective Antimicrobial stewardship (AMS) significantly reduces inappropriate antibiotic use and improves patient outcomes. In low-resource settings, AMS implementation may require concurrent strengthening of clinical microbiology capacity therefore additional investments. We assessed the cost-effectiveness of implementing AMS at Tikur Anbessa Specialised Hospital (TASH), a tertiary care hospital in Ethiopia.

Design We developed a Markov cohort model to assess the cost-utility of pharmacist-led AMS with concurrent strengthening of laboratory capacity compared with usual care from a 'restricted societal' perspective. We used a lifetime time horizon and discounted health outcomes and cost at 3\% annually. Data were extracted from a prospective study of bloodstream infections among patients hospitalised at TASH, supplemented by published literature. We assessed parameter uncertainty using deterministic and probabilistic sensitivity analyses. Setting Tertiary care hospital in Ethiopia, with 800 beds and serves over half a million patients per year. Population Cohort of adults and children inpatient population aged 19.8 years at baseline.

Intervention Laboratory-supported pharmacist-led AMS compared with usual care. Usual care is defined as empirical initiation of antibiotic therapy in the absence of strong laboratory and AMS.

Outcome measures Expected life-years, quality-adjusted life-years (QALYS), costs (US\$2018) and incremental costeffectiveness ratio.

Results Laboratory-supported AMS strategy dominated usual care, that is, AMS was associated with an expected incremental gain of 38.8 QALYs at lower expected cost (incremental cost savings:US\$82 370) per 1000 patients compared with usual care. Findings were sensitive to medication cost, infection-associated mortality and AMSassociated mortality reduction. Probabilistic sensitivity analysis demonstrated that AMS programme was likely to be cost-effective at $100 \%$ of the simulation compared with usual care at $1 \%-51 \%$ of gross domestic product/capita. Conclusion Our study indicates that laboratorysupported pharmacist-led AMS can result in improved health outcomes and substantial healthcare cost savings, demonstrating its economic advantage in a tertiary care hospital despite greater upfront investments in a lowresource setting.
Strengths and limitations of this study

- To our knowledge, this is the first study to investigate the cost-utility of laboratory supported antimicrobial stewardship (AMS) intervention for inpatients in Africa.

- A Markov cohort model was developed based on currently available evidence and we used infectionrelated mortality to model AMS effectiveness, the recommended outcome measure.

- Most parameter inputs used in the model were collected on the Ethiopian setting, reflecting the situation in low-resource settings, but some data including utilities were derived from high-income countries as these were the only data available.

- Extensive sensitivity and scenario analyses were performed to assess parameter uncertainty.

- A limitation of this study is that it did not account for disease transmission dynamics, which likely underestimated the benefit of AMS; and did not consider reinfection as the healthcare system was not designed to trace such cases.

\section{INTRODUCTION}

Excessive and inappropriate use of antibiotics are key drivers of increasing antimicrobial resistance globally, contributing to substantial health and economic losses. ${ }^{12}$ Multidisciplinary antimicrobial stewardship (AMS) is one of the most effective strategies to optimise antibiotic use, prevent medication errors, reduce healthcare expenditure and curb resistance, ${ }^{34}$ even in low-income and middle-income countries as described in a recent systematic review. ${ }^{5}$ However, additional investments, particularly in human resources and laboratory infrastructure, presents a challenge to AMS implementation in low-resource settings. ${ }^{6}$

Few economic evaluations of AMS programmes have been conducted to date; systematic reviews of these studies found AMS to be cost-effective in high and mid-resource 
settings, but none of the studies analysed cost-effectiveness in a low-resource setting like Ethiopia. ${ }^{78}$

Bloodstream infection (BSI), particularly among patients with a hospital-associated infection, is a major cause of in-hospital mortality. ${ }^{9}$ Between October 2016 and October 2018, the majority of BSI $(94.5 \%)$ at Tikur Anbessa Specialised Hospital (TASH), Ethiopia's largest referral and teaching hospital, were hospital associated. Enterobacterales accounted for $49.4 \%$ of the organisms isolated from blood cultures (Staphylococcus aureus, Pseudomonas spp and Acinetobacter spp were isolated in 35\%, $12 \%$ and $4.6 \%$, respectively; other organisms were identified in $13.5 \%$ of cases). More than $70 \%$ of Enterobacterales were resistant to third-generation cephalosporins (extended spectrum beta-lactamase producers). Amikacin and meropenem were the only two antibiotics to which more than $70 \%$ of Gram-negative blood isolates remained susceptible, but resistance to carbapenems was noted in $18 \%$ of Klebsiella spp and $10 \%$ of Escherichia coli isolates. The high burden of drug-resistant BSI was associated with widespread use of antibiotics. ${ }^{10}$ Inadequate investments in laboratory development and under-utilisation of pharmacists in the institution intensified inappropriate prescriptions of antibiotics. ${ }^{11} 12$

We recently demonstrated the feasibility and impact of a laboratory intervention aimed at informing therapeutic decisions through basic but adequate microbiological workup. ${ }^{12}$ Before beginning a laboratory-strengthening intervention, we conducted a need assessment and found that the bacteriology laboratory was under-resourced, resulting in inadequate infrastructure, varying staff proficiency, lack of essential supplies such as reagents to conduct key tests and lack of standardisation. ${ }^{12}$ Moreover, clinicians' distrust of laboratory results coupled with poor collaborative relationship with laboratory personnel contributed to the laboratory's underutilisation. ${ }^{13}$ As a result, we initiated a laboratory-strengthening intervention to address these underlying problems. We were able to build a reasonable-quality bacteriology laboratory as a result of the intervention, which resulted in significant increases in utilisation. In the first 18 months of the implementation, the number of specimens analysed grew from 15 per day to over 75 per day, and the number of blood cultures tested increased from an average of 2 per day to over 45 per day. ${ }^{12}$ We subsequently implemented a laboratory-supported pharmacist-led AMS programme, which led to a substantial reduction in antibiotic costs compared with the year prior. Cessation of this programme was immediately followed by a twofold increase in antibiotic consumption (days of therapy/1000 patient-days), as well as increased length of stay and in-hospital mortality. ${ }^{14}$ The antibiotic cost savings (19\% cost reduction for the institution) was observed during an intervention that was limited to $25 \%$ of hospitalised patients (in two wards), and were equivalent to laboratory reagent costs invested in the support of the bacteriology laboratory. However, the upfront investment to strengthen the microbiology component of the programme (eg, purchase of equipment such as an automated blood culture platform), costs associated with additional human resources and providing external expertise for training and other costs, are important concerns. ${ }^{12}{ }^{14}$ We, therefore, evaluated the cost-effectiveness of a laboratory-supported pharmacist-led AMS programme compared with usual care (no AMS and no specific laboratory intervention) in a low-resource setting tertiary teaching hospital in Ethiopia.

\section{METHODS}

We developed a Markov cohort model comparing the cost-utility of a laboratory-supported pharmacist-led AMS with usual care at TASH in Ethiopia, from a 'restricted societal' perspective. The 'restricted societal' perspective includes all direct medical costs but not productivity loss or other costs, taking into account Ethiopia's healthcare financing mechanism (online supplemental data supplement 1), which comprises multiple sources including: the patient, public funding, donors, public or private insurance and/or other civic societies. Accordingly, all direct medical costs paid by any party (eg, medication cost, investigation/procedural cost, microbiology/culture and sensitivity test cost, staff time cost, admission and other hospitalisation costs) were included. Outcomes are expected life years, quality-adjusted life-ears (QALY) and costs (US\$2018) over a lifetime time horizon, and the incremental costeffectiveness ratio (ICER) expressed as cost per QALY gained. We discounted costs and QALYs at an annual rate of $3 \%$ as recommended by WHO ${ }^{15}$ Cost-effectiveness was assessed against commonly used thresholds level of 1-3× gross domestic product (GDP)/ capita $^{15}$ and $1 \%-51 \%$ of GDP/capita. ${ }^{16}$ The GDP per capita in Ethiopia at the time of the study was US $\$ 873 .{ }^{17}$

\section{Study setting}

The study was conducted at TASH, the largest teaching hospital in Addis Ababa, Ethiopia. The hospital has 800 operational beds and serves over half a million patients per year (330000 outpatients and 20000 inpatients, with an average length of stay of 9.3 days). The hospital is staffed with 1059 physicians of which four had expertise in infectious diseases at the time of the study; 80 pharmacists and seven microbiology laboratory technologists. The AMS intervention was implemented in medicine and paediatric wards.

\section{Description of usual care and AMS intervention Usual care}

Usual care is defined as empirical initiation of antibiotic therapy in the absence of strong clinical microbiology and AMS. A formal infectious diseases specialist consultation could be requested as part of hospital services, but pharmacists' tasks were limited to dispensing practice and they had no clinical pharmacy role in hospital wards. 


\section{Laboratory-supported pharmacist-led AMS Intervention}

The laboratory-supported AMS intervention has extensively been described elsewhere, but briefly, consisted of strengthening the capacity of the bacteriology diagnostic laboratory ${ }^{10}$ and implementing a stewardship programme on selected wards. ${ }^{14}$ A major focus of the laboratory strengthening intervention was on performing blood culture testing routinely for all hospitalised patients with fever or signs of sepsis, to enable reliable diagnoses of BSIs and detection of antimicrobial resistance; neither of which were possible in usual care. The AMS intervention was limited to selected wards but focused on duration of antibiotic therapy and pathogen-specific antibiotic choices when culture results were available. Compared with usual care when virtually all antibiotic courses were empiric and consisted of multiple antibiotics for prolonged duration, the AMS team recommended to change or discontinue over half of the antibiotics prescribed, leading to shorter courses of therapy for patients without evidence of sepsis or BSI, and presumably more effective antibiotics for patients with microbiologically confirmed infections such as BSI.

\section{Model structure}

We constructed a Markov cohort model simulating the natural progression and clinical pathway of hospitalised patients at TASH. The model was built using TreeAge Software, Williamstown, Massachusetts USA. Figure 1 depicts the model structure. Patients either had or did not have a BSI. Those admitted without BSI could acquire BSI during their hospital stay. Patients with BSI may die from infection, progress to sepsis or improve and be discharged. Because current sepsis definitions that rely on the quick Sequential Organ Failure Assessment score ${ }^{18}$

A
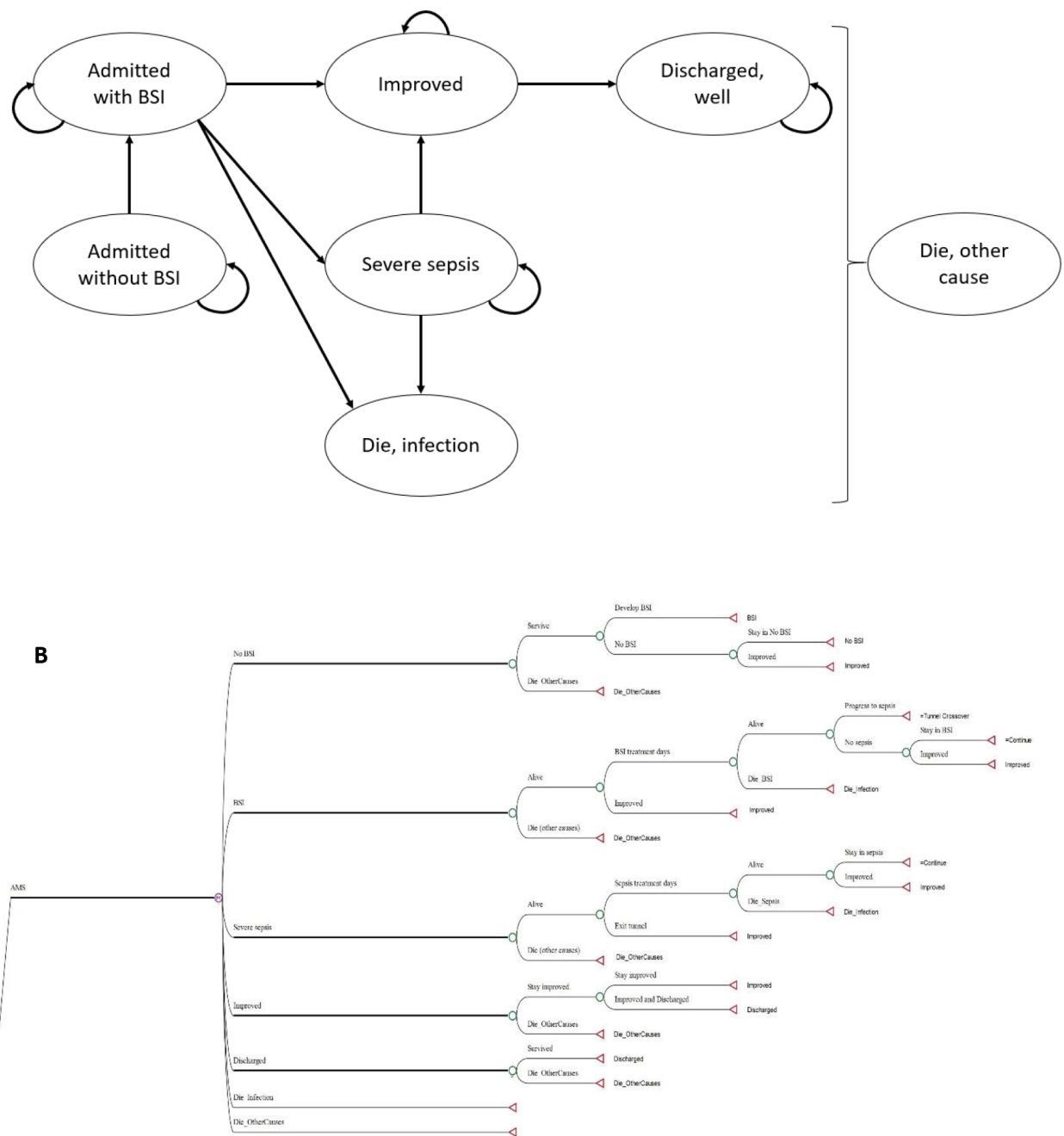

Figure 1 Schematic presentation of health state model. (A.) Markov state transition diagram of inpatients with bloodstream infections. The seven circles show possible conditions or health states while the arrows imply transitions of patients among different health states. (B.) illustrative structural model of an AMS intervention strategy. The usual care strategy also has the same model structure, but with varied input values. During each cycle, individuals could be in any of the seven health states and the transitional probabilities determine their possible transition between health states. A chance node (circle) denotes the alternative possibilities or possible outcomes, whereas a terminal node (triangle) marks the endpoint of a scenario. AMS, antimicrobial stewardship; BSI, bloodstream infection. 
Table 1 Model parameters point estimate values and ranges

\begin{tabular}{|c|c|c|c|}
\hline Parameters & Base-case & Range & Reference \\
\hline \multicolumn{4}{|l|}{ Probabilities } \\
\hline Bloodstream infection at admission & 0.05 & $0.04-0.06$ & 1021 \\
\hline Progression to sepsis & 0.20 & $0.12-0.26$ & $21-24$ \\
\hline $\begin{array}{l}\text { Bloodstream infection-associated mortality, usual } \\
\text { care }\end{array}$ & 0.31 & $0.058-0.29$ & 10 \\
\hline $\begin{array}{l}\text { Bloodstream infection-associated mortality } \\
\text { reduction, AMS }\end{array}$ & 0.06 & $0.00-0.32$ & 41 \\
\hline \multicolumn{4}{|l|}{ Health-related utilities } \\
\hline Well & 0.74 & $0.48-0.83$ & $31-34$ \\
\hline \multicolumn{4}{|l|}{ Costs (US\$) } \\
\hline Cost of blood culture test, per patient & US\$8 & US\$6-US\$24 & TASH, 2018 \\
\hline AMS staff time cost, per patient & US\$3 & US\$1-US\$6 & TASH, 2018 \\
\hline Daily hospitalisation cost, per patient & US\$5 & US\$1-US\$35 & TASH, 2018 \\
\hline $\begin{array}{l}\text { Bloodstream treatment cost per hospital stay per } \\
\text { patient, usual care }\end{array}$ & US\$1872 & US\$255-US\$2821 & TASH, 2018 \\
\hline Bloodstream treatment cost per hospital stay, AMS & US\$289 & US\$255-US\$2821 & TASH, 2018 \\
\hline $\begin{array}{l}\text { Total cost of automated blood culture platform } \\
\text { including consumables and technologist training } \\
\text { cost }\end{array}$ & US\$97464 & US $\$ 80000-U S \$ 150000$ & SLP, 2018 \\
\hline
\end{tabular}

AMS, antimicrobial stewardship; SLP, Setema Limited Plc; TASH, Tikur Anbessa Specialised Hospital.

lack sensitivity among patients treated outside of intensive care units and few data exist on its ability to predict BSI, ${ }^{19}$ we used the systemic inflammatory response syndrome criteria to define sepsis. ${ }^{20}$ Patients with severe manifestations of sepsis could die from infection or improve and be discharged. Patients could die from other causes (ie, not related to BSI) at any time. The simulated population reflects the TASH patient population, comprising children and adults with a mean age of 19.8 years (ranged from 15 days to 78 years with SD of 18.6 years). Patients were followed in daily time steps (ie, 1-day cycle length) over their life expectancy. A half-cycle correction was done to assume that events occur halfway through a cycle (rather than at the beginning of each cycle).

\section{Parameter inputs}

All data including programme effectiveness, probabilities, costs and utilities are summarised in table 1.

\section{Probabilities}

Probability estimates were obtained from our primary study, supplemented by the literature (table 1). Data from the literature were assessed by a five-member expert team comprising clinicians and pharmacists with significant experience in infectious diseases for relevance and applicability to our setting. The prevalence and incidence of hospital acquired infections in low-resource settings were estimated to be 15.5 (12.6-18.9) and 7.4 (4.4-12.2) per 100 patients-days, respectively. ${ }^{21}$ BSI accounted for $30 \%$ of all infections in our prospective study. ${ }^{10}$ The probabilities of patients admitted with BSI and acquiring BSI in hospital were assumed to be similar for both strategies, that is, we assume that AMS has no impact on in-hospital acquisition of disease. BSI-associated 14-day mortality was $31 \%,{ }^{10}$ while $20 \%$ of BSI patients may develop sepsis. ${ }^{22}$ We assumed progression to sepsis to be similar for both strategies with the assumption that AMS effectiveness on disease progression is negligible. The 28-day mortality from patients with severe manifestations of sepsis was $40 \% \cdot{ }^{2324}$ According to our hospital database, mean annual all-cause in-hospital mortality was $5.8 \%$. We used life expectancy data from the WHO Global Health Observatory life table data repository for Ethiopia to compute agespecific probabilities of in-hospital and posthospitalisation deaths unrelated to the current infection. ${ }^{25}$ Since rates of events are defined over a range of observation periods, we converted them to daily probabilities to match the model's cycle length. Assuming events occur at constant rate $r$ per unit time $t$, we can convert a rate into a probability and vice versa. ${ }^{26}$ For example, given a $40 \%$ mortality rate of sepsis in 28 days; we converted it into daily death 
rate $(-[\ln (1-0.4)] / 28)=0.018244)$, and then calculated the daily probability $(1-\exp (1-0.018244)=0.018078$.

\section{Utilities}

Utility weights were derived from the literature. The utility score for patients with BSI was derived from Lee et al. ${ }^{27}$ Utilities for patients without BSI and patients improved from infection but hospitalised for other reasons were considered to be the same as utilities for general medical inpatients and were obtained from a mixed population of patients in a teaching hospital in England ${ }^{28}$ and a review of utilities by Tengs and Wallace study. ${ }^{29}$ The utility for patients with severe manifestations of sepsis obtained from Hofhuis et al study was $0.45^{30}$ and utility for survivors from infection was $0.74 .^{31-34}$ Due to lack of utilities for patients in low-income and middle-income countries, we used utilities from higher-income countries since the general population mean utility for Ethiopia (EQ-5D index $=0.94$ ) was found to be comparable to high-income countries. ${ }^{35-37}$ We assumed that surviving patients have the same life expectancy as the general population but with lower than average quality of life. ${ }^{38}$

\section{Cost}

All cost data were obtained from our prospective, quasiexperimental study assessing the impact of a weekly audit-feedback AMS intervention and hospital records. We estimated the mean hospitalisation cost per day to be US\$5 per patient, excluding medication and microbiology work-up costs to avoid double counting. Mean total treatment cost per hospital stay in the AMS strategy was US\$289 per patient while patients in usual care incurred US $\$ 1872$. The large cost reduction during the AMS intervention was primarily due to: (1) changing the large volume of broad-spectrum prescriptions to narrowspectrum (generally less costly) antibiotics; (2) significantly reducing treatment duration and (3) frequent discontinuation of incorrectly/unnecessarily prescribed expensive antibiotics (eg, vancomycin, which accounted for $30 \%$ of the antibiotics budget recommended to be discontinued in $60 \%$ of cases with good acceptance). ${ }^{14}$ Patient with severe manifestations of sepsis had additional daily costs of US $\$ 34$ for AMS and US $\$ 144$ for usual care. The cost of each blood culture and sensitivity test including microbiology technologist full-time equivalent cost was US $\$ 8$. We received an in-kind donation from bioMérieux for a BacT/ALERT 3D 120 Combo instrument (bioMérieux, France). This one-time upfront implementation cost of the automated blood culture platform worth $\$ 70740$ and its useful life was assumed to be 8 years (5-15 years). We used a straight-line method of depreciation that assumes the usefulness of the asset is the same over the entire life of the asset to calculate the per cycle cost. In addition, we spent US $\$ 26200$ per year on blood culture bottles and consumables, US $\$ 1900 /$ year for an institution-wide subscription to an electronic application displaying empiric treatment guidelines and cumulative antibiogram accessible to end-users, as well as US\$524/ year for six microbiology laboratory technologists to access an online proficiency testing resource. The fulltime equivalent cost for the AMS team was calculated by multiplying the mean professional time spent per patient by their pay-scale salaries for infectious disease physicians and pharmacists with more than 5 years work experience. Average AMS staff time cost was calculated to be US $\$ 3$ per patient. The continuous support of Canadian microbiologists (a total of 50 visit-days per year) was essential for the successful implementation of the programme but excluded from the study as this implementation mode may not be the case for most hospitals in Ethiopia. All costs are expressed in 2018 US $\$$ adjusted for inflation using the average 2018 consumer price index ${ }^{39}$ and converted to US\$ using the mean 2018 exchange rate of 27.6 birr per US $\$ 1{ }^{40}$

\section{Effectiveness}

Effectiveness estimates were based on our prospective quasi-experimental study assessing the effectiveness of implementing AMS, supplemented by the literature. In our prospective study, AMS was effective in reducing treatment duration by 4.5 days ( 8.5 vs 13 days). Regardless of the specific antibiotic regimen, reduction in BSIassociated mortality due to AMS intervention was reported to be $6 \%{ }^{41}$ and we assumed the same mortality reduction for patients with severe manifestations of sepsis (table 1). Generalisability of previous AMS cost-effectiveness studies was limited owing to heterogeneity of effectiveness measures across studies which is ascribed to the wider scope of clinical benefits that extend from the intervened patient to deterrence of forthcoming infections caused by multidrug resistant pathogens. ${ }^{38} 4243$ Recent studies recommended future economic evaluations of AMS to be pathogen or disease specific (eg, BSI) ${ }^{84}$ We targeted BSI because it was an infectious disease that poses a major burden at the hospital. Also, the use of BSI-associated mortality as an outcome measure was suggested since the highest impact of inappropriate antibiotic therapy on death, which otherwise can be substantially reduced by AMS, was observed in patients with BSI. ${ }^{44}$ Furthermore, using mortality is an important component of QALYs, which incorporate quantity and quality of life, making it possible to compare health-related interventions with widely varying outcomes across different areas. ${ }^{8}$

Participants written informed consent was not required in accordance with the national and institutional guidelines.

\section{ANALYSIS}

\section{Base-case analysis}

We defined the base-case analysis as: mean age at admission of 19.8 years and 14-days BSI-associated probability of death for usual care was 0.31 , with a $6 \%$ relative reduction for the laboratory-supported pharmacist-led AMS strategy. 


\section{Scenario analysis}

We conducted two different scenario analyses on the basis that the large impact of AMS in the first year postintroduction would not be sustainable over a longer time period.

In scenario 1, we assumed no effectiveness of AMS on mortality, a similar distribution of expensive and inexpensive medication in AMS and usual care, excluding the initial investment to build microbiology capacity (ie, assuming strong microbiology facility already exists) and an average treatment duration of 9 days for the AMS strategy (vs 12 days in usual care).

In scenario 2, we consider the same inputs as scenario 1, except the reduction of treatment days with AMS was only 1 day and the initial cost of microbiology was considered as an implementation cost.

\section{Sensitivity analysis}

We performed deterministic and probabilistic sensitivity analyses to assess parameter uncertainty. Deterministic (one-way) sensitivity analysis allows for each input variable to vary within a range of values. We also performed probabilistic sensitivity analysis by simultaneously varying all variable inputs across their parameter value distribution using 10000 Monte Carlo simulations. The ranges of values for costs and duration of therapy were obtained from our prospective study. Utility and probability ranges were obtained from the literature. We used gamma distributions for costs and treatment days, and beta distributions for probabilities and utilities.

\section{Patient and public involvement}

No patient involved.

\section{RESULTS}

\section{Base-case analysis}

The discounted and undiscounted health outcomes (life-years and QALYs), costs and ICER for laboratorysupported pharmacist-led AMS compared with usual care are presented in table 2. In the base-case analysis, AMS was the dominant strategy, that is, more effective and less costly than usual care. The total expected life years (LYs) gained were 17.9 (28.6 LYs undiscounted) per patient with AMS strategy and 17.9 LYs (28.5 LYs undiscounted) per patient in the usual care. AMS strategy generated a mean of 13.2772 expected QALYs (21.2807 QALYs undiscounted) compared with 13.2384 QALYs (21.2185 QALYs undiscounted) with usual care. The total expected mean cost per patient were US $\$ 42.0$ for AMS and US $\$ 124.37$ for usual care. Thus, AMS resulted in an incremental gain of 52.2 LYs (83.6 LYs undiscounted) per 1000 admissions,

Table 2 Base-case and scenario analysis results for AMS versus usual care in Ethiopia

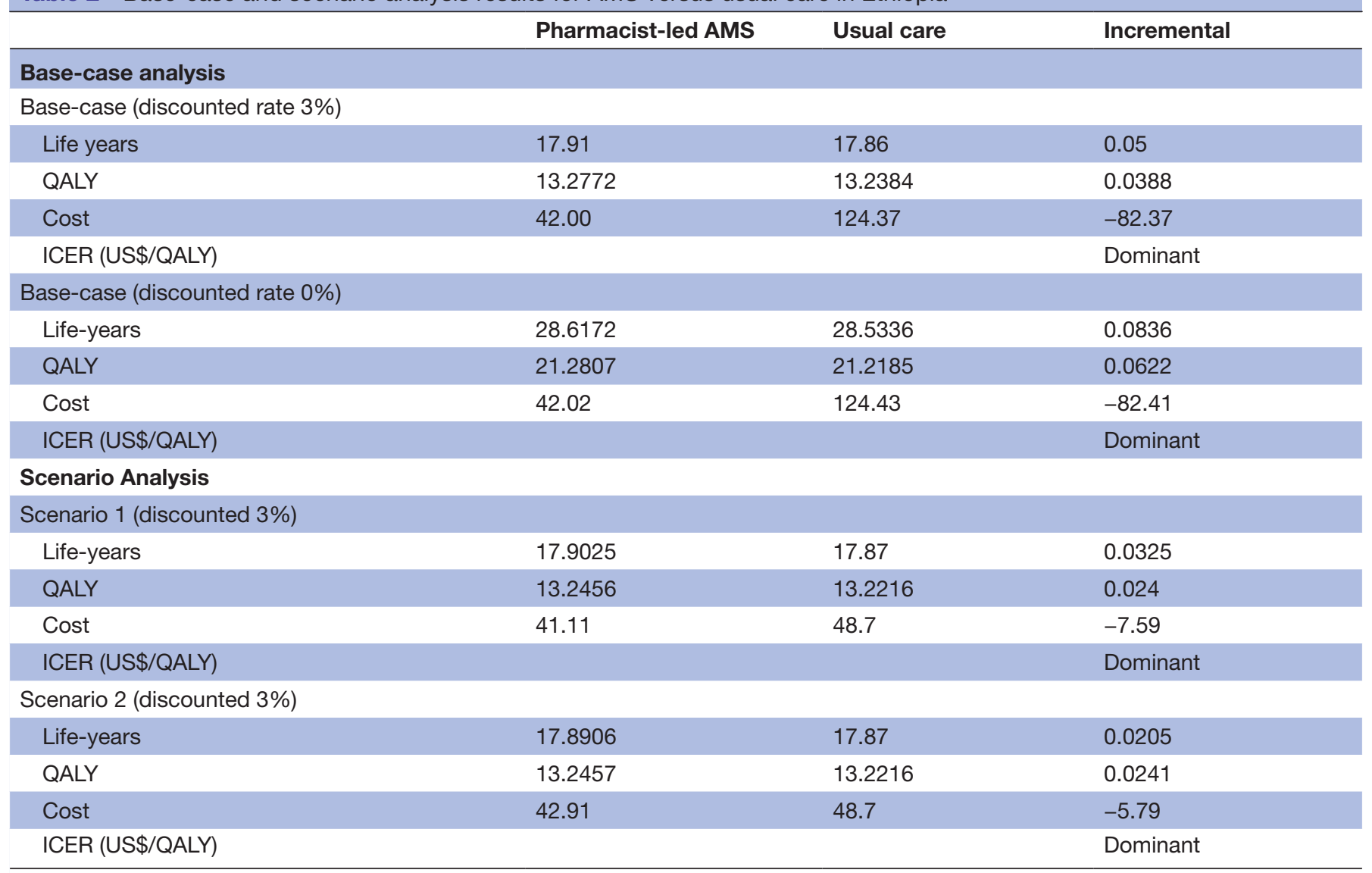

AMS, antimicrobial stewardship; ICER, incremental cost-effectiveness ratio; QALY, quality-adjusted life-year. 


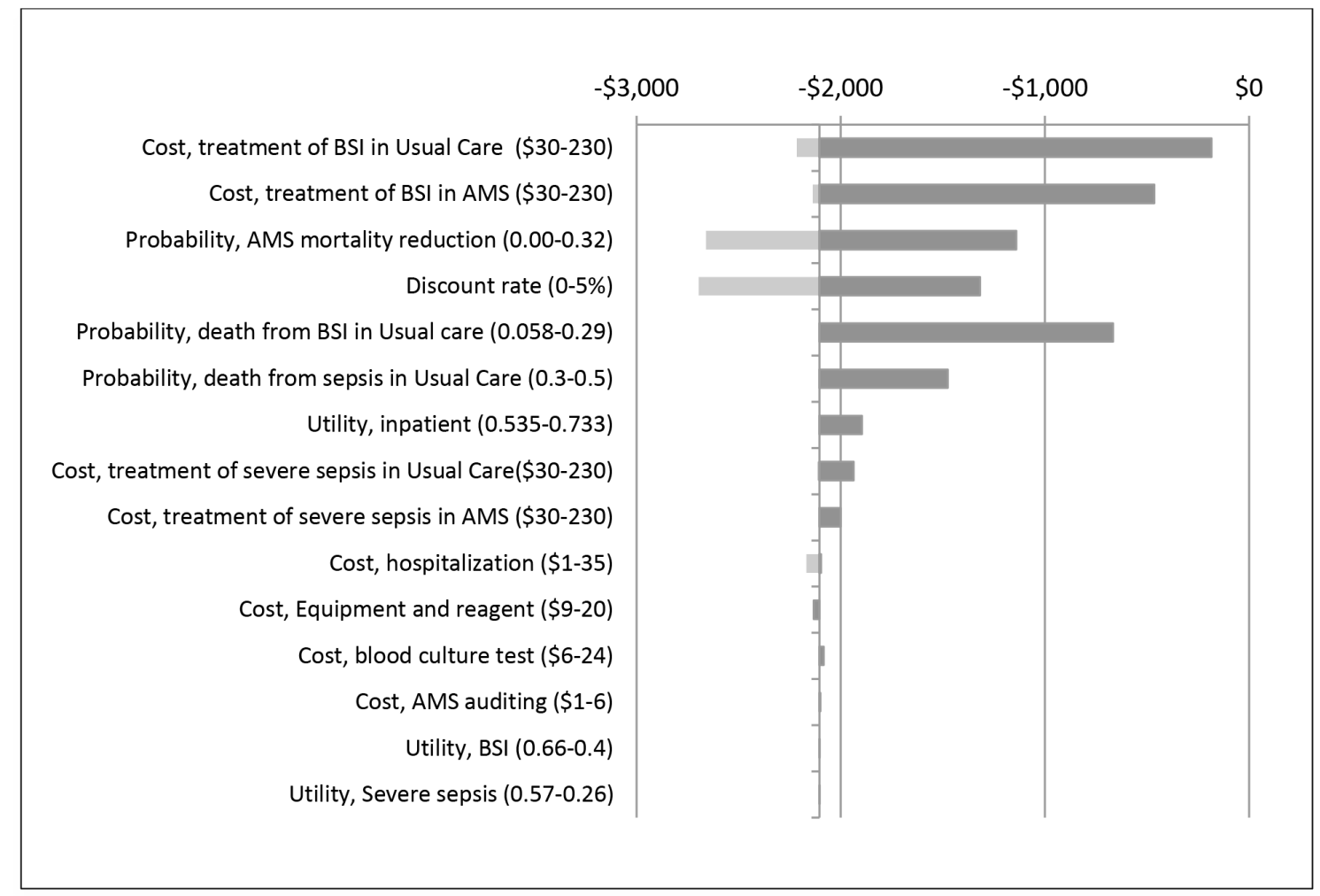

Figure 2 Tornado diagram showing the sensitivity analysis for the base-case scenario (discounted at $3 \%$ ). The vertical line represents the base-case incremental cost-effectiveness ratio (ICER) for AMS and the x-axis shows the range of the ICER when the base-case values are varied (ranges shown in brackets). The negative ICER means AMS intervention is a dominant strategy (less costly and better health outcome). AMS, antimicrobial stewardship; BSI, bloodstream infection.

translating to 38.8 QALY gain (62.2 QALY undiscounted), while saving US\$82370 per 1000 patients (US\$82410 undiscounted).

\section{Scenario analysis}

In the scenario analysis, laboratory-supported pharmacist-led AMS was the dominant strategy for both scenarios (table 2).

\section{Deterministic sensitivity analysis}

Deterministic sensitivity analysis for the base-case scenario showed AMS to be dominant compared with usual care across plausible ranges for all variables tested. The most influential variables which increased the ICER significantly were treatment costs, BSI-associated mortality, discount rate and mortality reduction achieved with AMS implementation (figure 2).

\section{Probabilistic sensitivity analysis}

Probabilistic sensitivity analysis (figure 3) shows AMS intervention to be the dominant strategy in $100 \%$ of simulations at a threshold level of $1 \times \mathrm{GDP} /$ capita and $1 \%-51 \%$ of GDP per capita.

\section{DISCUSSION}

Although the causes of antimicrobial resistance are multifaceted, it is predominantly linked to excessive and inappropriate use of antibiotics and is a growing problem in Ethiopia and other low-income and middleincome countries. ${ }^{210}{ }^{45}$ In this study, we demonstrate that a laboratory-supported pharmacist-led AMS intervention was dominant compared with usual care, with an expected 0.0388 QALYs gained and cost savings of US $\$ 82.37$ per patient. This translates to 38.8 QALYs gained and total cost savings of US\$82370 per 1000 patient admissions. Findings were robust to assumptions made, sensitivity analysis did not identify any parameter thresholds at which the conclusion would change, and ICER values were well below the commonly used cost-effectiveness threshold level of $1 \times \mathrm{GDP} /$ capita $^{15}$ and $1 \%-51 \%$ of GDP/capita. ${ }^{16}$ Our findings are consistent with other studies from highincome countries: AMS implementation was found to be cost-effective in hospitals in the USA, Australia and European countries. ${ }^{8846}$

The cost savings obtained by the intervention are mainly due to the reduction of antibiotic use (shorter treatment duration, discontinuation of unnecessary antibiotics and reduced duration of hospitalisation). ${ }^{14}$ However, it is important to recognise that these large cost savings were obtained in the first year of the intervention. Before initiating this intervention, very costly antibiotics (vancomycin, meropenem, third-generation and fourthgeneration cephalosporins) were widely prescribed regardless of the source and suspected etiological agents of infection. In our previous study, we found that vancomycin alone accounted for $30 \%$ of the total antibiotic 


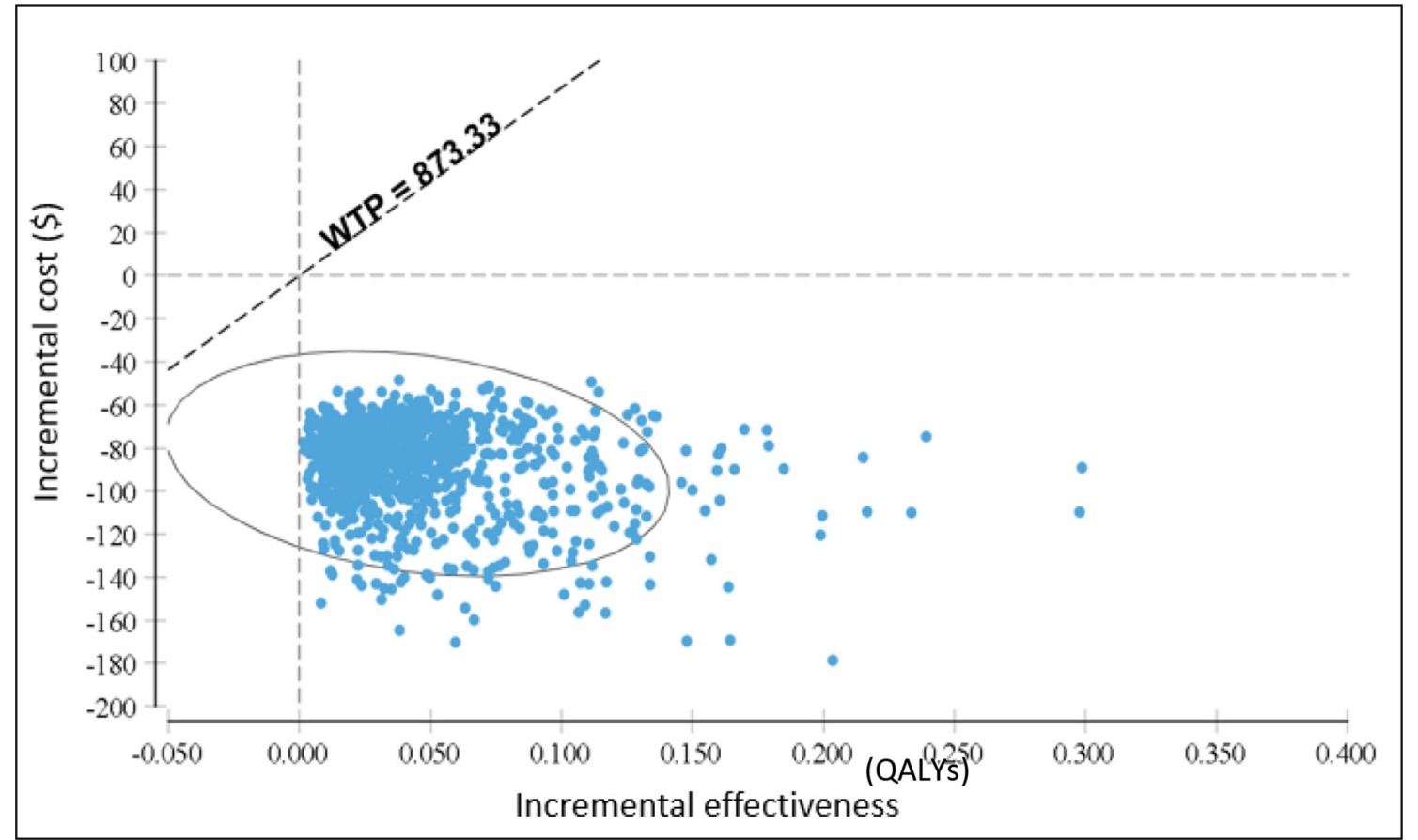

Figure 3 Base-case results from probabilistic sensitivity analysis: cost-effectiveness scatter plot of simulated incremental cost and incremental effectiveness for AMS compared with usual care, at a 3\% annual discount rate. The ellipsis contains $95 \%$ of cost-effectiveness estimates. AMS, antimicrobial stewardship; QALYs, quality-adjusted life-years; WTP, willingness to pay.

spending and four antibiotics (vancomycin, meropenem, ceftazidime and ceftriaxone) accounted for $66 \%$ of the total antibiotics budget. ${ }^{11} \mathrm{~A}$ one-gram vial of vancomycin, meropenem, ceftazidime and ceftriaxone each cost US $\$ 9$, US $\$ 8$, US $\$ 6$ and US $\$ 3.5$; respectively. Indiscriminate use of these antibiotics in the preintervention period likely facilitated the dramatic reduction of antibiotic use in a short time period, but such a large effect might not be sustained over a longer time frame. Interestingly, AMS remained cost-effective in the scenario analysis where there was no significant impact on mortality and only minimal impact on antibiotic use with AMS. On the other hand, we did not account for the long-term impact of AMS in reducing antimicrobial resistance and its associated costs, potentially underestimating the costeffectiveness of AMS. We also anticipate that strengthening clinical microbiology has additional benefits that we did not account for in this study.

Our study has some limitations. First, we did not consider reinfection because the referral system in place does not allow direct readmission to specialised hospitals such as TASH. Second, we did not account for disease transmission dynamics. Because AMS shortens the hospital length of stay thereby decreasing in-hospital transmission risk, omission of transmission dynamics might have underestimated the benefit (more cost-effectiveness) of AMS. Further, the scope of our economic evaluation was limited to the impact of AMS in BSI patients and we did not consider the effects of AMS on early or timely initiation of antibiotics. Delaying initiation of appropriate antibiotic therapy is significantly associated with early death, independent of pathogen type, severity, portal of entry and type of settings. ${ }^{47}$ Future assessments should extend the scope and measure the effect of early involvement of AMS team on the management of patients with infectious diseases in low-resource settings. We recommend future forthcoming economic evaluations of AMS to include other public health-relevant infectious diseases, as a variety of other infectious diseases can be effectively addressed by AMS intervention. Finally, due to the scarcity of country specific data, several probabilities and all utility inputs were obtained from high-income settings.

To the best of our knowledge, this is the first comprehensive cost-utility analysis using a Markov model assessing AMS intervention for inpatients in Africa. Laboratory-supported pharmacist-led AMS interventions in low-resource settings result not only in significant clinical benefits to individual patients, but are economically advantageous. Substantial savings in healthcare costs can be achieved, even accounting for upfront investments in equipment and training. These findings should guide improvements in the standards of healthcare for lowresource settings.

\section{CONCLUSIONS}

Our analysis suggested that the implementation of a laboratory-supported pharmacist-led AMS programme at a tertiary care hospital in a low-resource setting was dominant (saving costs and improving health) compared with usual care. Our findings support implementation of AMS in similar settings. 
Author affiliations

${ }^{1}$ School of Pharmacy, College of Health Sciences, Addis Ababa University, Addis Ababa, Ethiopia

${ }^{2}$ Institute of Health Policy Management and Evaluation, University of Toronto, Toronto, Ontario, Canada

${ }^{3}$ Toronto Health Economics and Technology Assessment (THETA) Collaborative, University Health Network, Toronto, Ontario, Canada

${ }^{4}$ School of Public Health, College of Health Sciences, Addis Ababa University, Addis Ababa, Ethiopia

${ }^{5}$ Pediatrics and Child Health, Pediatric Cardiology Unit, College of Health Sciences, Addis Ababa University, Addis Ababa, Ethiopia

${ }^{6}$ St Paul's Hospital Millennium Medical College, Addis Ababa, Ethiopia

${ }^{7}$ American Medical Center, Specialty Center for Infectious Diseases and Travel Medicine, Addis Ababa, Ethiopia

${ }^{8}$ School of Medicine, Addis Ababa University College of Health Sciences, Addis Ababa, Ethiopia

${ }^{9}$ J.D. MacLean Centre for Tropical Diseases, McGill University Health Centre, Montreal, Québec, Canada

${ }^{10}$ Divisions of Infectious Diseases and Medical Microbiology, McGill University Health Centre, Montreal, Québec, Canada

Twitter Gebremedhin Beedemariam Gebretekle @Gebremedhinb and Stephen Mac @_StephenMac

AcknowledgmentsThe authors would like to thank Addis Ababa University for sponsoring the PhD candidate (author GBG), McGill University for providing financial support for implementing the laboratory intervention and the AMS programme, and the Canada Research Chair in Economics of Infectious Diseases for financing the economic evaluation study. We also would like to thank the Toronto Addis Ababa Academic Collaboration (TAAAC) and AIDSFree Project, USAID-Ethiopia for providing financial support for the candidate to attend relevant courses at the Toronto Health Economics and Technology Assessment (THETA) Collaborative, University of Toronto, Canada. Finally, we would like to extend our gratitude to Professor Heather Boon and Dr. Clare Pain for their support to GBG.

Contributors GBG performed literature search, construct the model, conducted the economic analysis and drafted the manuscript. BS supervised and guided the economic analysis. SM contributed to the economic analysis. GBG, DHM, SM, TGF and BS designed the study. GBG, TGF and BS solicited the funding for the economic analysis. SM and CPY solicited the funding for AMS and laboratory interventions and SM and WA supervised the implementation of the primary studies from which GBG, WA and TA acquired the data. ML and WAD contributed to the implementation of laboratory and AMS interventions. BS provided administrative, and technical support. DHM, SM, TGF and BS supervised the economic analysis. All authors contributed to data interpretation and approved the final manuscript. GBG accepts full responsibility for the finished work and/or the conduct of the study, had access to the data, and controlled the decision to publish.

Funding This work was supported by grants from the Research Institute of the McGill University Health Centre (GRANT \#974) under the auspices of the Addis Ababa University (AAU) and McGill Partnership for Infectious Diseases (AMP-ID: http://amp-id.org) and the Canada Research Chair in Economics of Infectious Diseases held by Beate Sander (CRC-950-232429). In-kind support from bioMérieux was provided via an investigator-initiated research grant. GBG is sponsored by Addis Ababa University for his Ph.D. studies. CPY holds a 'Chercheurboursier clinicien' career award from the Fonds de recherche du Québec-Santé (FRQS).

Disclaimer The funder of the study had no role in study design, implementation, data analysis and interpretation.

Competing interests All authors declare that they have no competing interests. Patient consent for publication Not required.

Ethics approval This study was approved by the Institutional Review Board of the College of Health Sciences, Addis Ababa University, Ethiopia (Protocol Number: 045/15/IM), and the Research Institute of the McGill University Health Centre (study 15-178 MUHC).

\section{Provenance and peer review Not commissioned; externally peer reviewed.}

Data availability statement All data inputs relevant to the study are included in the article. The TreeAge * ${ }^{*}$ TREX file is available on request to the authors.

Supplemental material This content has been supplied by the author(s). It has not been vetted by BMJ Publishing Group Limited (BMJ) and may not have been peer-reviewed. Any opinions or recommendations discussed are solely those of the author(s) and are not endorsed by BMJ. BMJ disclaims all liability and responsibility arising from any reliance placed on the content. Where the content includes any translated material, BMJ does not warrant the accuracy and reliability of the translations (including but not limited to local regulations, clinical guidelines, terminology, drug names and drug dosages), and is not responsible for any error and/or omissions arising from translation and adaptation or otherwise.

Open access This is an open access article distributed in accordance with the Creative Commons Attribution Non Commercial (CC BY-NC 4.0) license, which permits others to distribute, remix, adapt, build upon this work non-commercially, and license their derivative works on different terms, provided the original work is properly cited, appropriate credit is given, any changes made indicated, and the use is non-commercial. See: http://creativecommons.org/licenses/by-nc/4.0/.

ORCID iD

Gebremedhin Beedemariam Gebretekle http://orcid.org/0000-0002-2485-505X

\section{REFERENCES}

1 O'Neill J. Antimicrobial Resistance : Tackling a Crisis for the Health and Wealth of Nations. The Review on Antimicrobial Resistance, Chaired by Jim O'Neill. Available: https://amr-review.org/ Publications.html

2 Bell BG, Schellevis F, Stobberingh E, et al. A systematic review and meta-analysis of the effects of antibiotic consumption on antibiotic resistance. BMC Infect Dis 2014;14:1-25.

3 Society for Healthcare Epidemiology of America, Infectious Diseases Society of America, Pediatric Infectious Diseases Society. Policy statement on antimicrobial stewardship by the Society for healthcare epidemiology of America (SheA), the infectious diseases Society of America (IDSA), and the pediatric infectious diseases Society (PIDS). Infect Control Hosp Epidemiol 2012;33:322-7.

4 Schuts EC, Hulscher MEJL, Mouton JW, et al. Current evidence on hospital antimicrobial stewardship objectives: a systematic review and meta-analysis. Lancet Infect Dis 2016;16:847-56.

5 Van Dijck C, Vlieghe E, Cox JA. Antibiotic stewardship interventions in hospitals in low-and middle-income countries: a systematic review. Bull World Health Organ 2018;96:266-80.

6 Cox JA, Vlieghe E, Mendelson M, et al. Antibiotic stewardship in low and middle-income countries: the same but different? Clin Microbiol Infect 2017:23:812-8.

7 Ibrahim NH, Maruan K, Azzuar H. Economic Evaluations on Antimicrobial Stewardship Programme : A Systematic Review Description of Included Studies. J Pharm Pharm Sci 2017;20:397-406.

8 Naylor NR, Zhu N, Hulscher M, et al. Is antimicrobial stewardship cost-effective? A narrative review of the evidence. Clin Microbiol Infect 2017;23:806-11.

9 Goto M, Al-Hasan MN. Overall burden of bloodstream infection and nosocomial bloodstream infection in North America and Europe. Clin Microbiol Infect 2013;19:501-9.

10 Semret M, Abebe W, Kong LY, et al. Prolonged empirical antibiotic therapy is correlated with bloodstream infections and increased mortality in a tertiary care hospital in Ethiopia: bacteriology testing matters. JAC Antimicrob Resist 2020;2:1-8.

11 Gebretekle GB, Haile Mariam D, Abebe W, et al. Opportunities and barriers to implementing antibiotic stewardship in low and middleincome countries: lessons from a mixed-methods study in a tertiary care hospital in Ethiopia. PLoS One 2018;13:e0208447-15.

12 Yansouni CP, Seifu D, Libman M, et al. A feasible LaboratoryStrengthening intervention yielding a sustainable clinical bacteriology sector to support antimicrobial stewardship in a large referral hospital in Ethiopia. Front. Public Health 2020;8:1-10.

13 Alemkere G, Gilagil G, Gebrehiwot T, et al. Physicians' utilization of microbiologic reports and determinants of their preference to order culture in Tikur Anbessa Specialized Hospital, Addis Ababa, Ethiopia. BMC Res Notes 2018;11:1-6.

14 Gebretekle GB, Haile Mariam D, Abebe Taye W, et al. Half of prescribed antibiotics are not needed: a pharmacist-led antimicrobial stewardship intervention and clinical outcomes in a referral hospital in Ethiopia. Front Public Health 2020;8:1-11.

15 WHO. Making choices in health: who guide to cost-effectiveness analysis. Geneva: World health organisation, 2003. Available: https:// apps.who.int/iris/handle/10665/42699

16 Woods B, Revill P, Sculpher M, et al. Country-Level costeffectiveness thresholds: initial estimates and the need for further research. Value Health 2016;19:929-35. 
17 Bank W, per capita GDP. current US\$): World Bank National Accounts Data and OECD National Accounts data files, 2017. Available: http://data.worldbank.org/indicator/NY.GDP.PCAP.CD

18 Singer M, Deutschman CS, Seymour CW, et al. The third International consensus definitions for sepsis and septic shock (sepsis-3). JAMA 2016;315:801-10.

19 Fernando SM, Tran A, Taljaard M, et al. Prognostic accuracy of the quick sequential organ failure assessment for mortality in patients with suspected infection. Ann Intern Med 2018;168:266-75.

20 Kaukonen K-M, Bailey M, Pilcher D, et al. Systemic inflammatory response syndrome criteria in defining severe sepsis. $N$ Engl $\mathrm{J} \mathrm{Med}$ 2015;372:1629-38.

21 Allegranzi B, Bagheri Nejad S, Combescure C, et al. Burden of endemic health-care-associated infection in developing countries: systematic review and meta-analysis. Lancet 2011;377:228-41.

22 Alberti C, Brun-Buisson C, Chevret S, et al. Systemic inflammatory response and progression to severe sepsis in critically ill infected patients. Am J Respir Crit Care Med 2005;171:461-8.

23 Lefrant J-Y, Muller L, Raillard A, et al. Reduction of the severe sepsis or septic shock associated mortality by reinforcement of the recommendations bundle: a multicenter study. Ann Fr Anesth Reanim 2010;29:621-8.

24 Stevenson EK, Rubenstein AR, Radin GT, et al. Two decades of mortality trends among patients with severe sepsis: a comparative meta-analysis*. Crit Care Med 2014;42:625-31.

25 WHO. Global Health Observatory data repository: Life tables by country - Ethiopia, 2018. Available: http://apps.who.int/gho/data/? theme $=$ main\& $\mathrm{vid}=60550$

26 Fleurence R, Hollenbeak C. Rates and probabilities in economic modelling. Pharmacoeconomics 2012;25:1-4 papers2://publication/ uuid/8850A6C3-6F44-4FA2-B3D9-F04ABC2769DE

27 Lee BY, Bailey RR, Smith KJ. Universal methicillin-resistant Staphylococcus aureus (MRSA) surveillance for adults at hospital admission: an economic model and analysis. Infect Control HospEpidemiol 2012;31:598-606.

28 Selai C, Rosser R. Eliciting EuroQol descriptive data and utility scale values from inpatients. A feasibility study. Pharmacoeconomics 1995;8:147-58

29 Tengs TO, Wallace A. One thousand health-related quality-of-life estimates. Med Care 2000;38:583-637.

30 Hofhuis JGM, Spronk PE, van Stel HF, et al. The impact of severe sepsis on health-related quality of life: a long-term follow-up study. Crit Care Trauma 2008;107:1957-64.

31 Edmond K, Dieye Y, Griffiths UK, et al. Prospective cohort study of disabling sequelae and quality of life in children with bacterial meningitis in urban Senegal. Pediatr Infect Dis J 2010;29:1023-9.

32 Heyland DK, Hopman W, Coo H, et al. Long-Term health-related quality of life in survivors of sepsis. short form 36: a valid and reliable measure of health-related quality of life. Crit Care Med 2000:28:3599-605.
33 Granja C, Dias C, Costa-Pereira A, et al. Quality of life of survivors from severe sepsis and septic shock may be similar to that of others who survive critical illness. Crit Care 2004;8:R91-8.

34 Honselmann KC, Buthut F, Heuwer B. Long-Term mortality and quality of life in intensive care patients treated for pneumonia and/ or sepsis: predictors of mortality and quality of life in patients with sepsis/pneumonia. J Crit Care 2015;30:721-6.

35 Devlin N, Shah K, Feng Y. Valuing health-related quality of life: an EQ-5D-5L value set for England. Health Econ 2017;27:1-16.

36 Welie AG, Gebretekle GB, Stolk E, et al. Valuing health state: an EQ-5D-5L value set for Ethiopians. Value in Health Regional Issues 2020;22:7-14.

37 M Versteegh M, M Vermeulen K, M A A Evers S, et al. Dutch tariff for the five-level version of EQ-5D. Value Health 2016;19:343-52.

38 Scheetz $\mathrm{MH}$, Bolon MK, Postelnick M, et al. Cost-Effectiveness analysis of an antimicrobial stewardship team on bloodstream infections: a probabilistic analysis. J Antimicrob Chemother 2009;63:816-25.

39 CSA. Country and regional level consumer price indices. fed. Demoratic Repub. Ethiop. Natl. plan. Comm. Centarl STAT. agency (CSA), 2018. Available: http://www.csa.gov.et/price-indices/ consumer-price-index/category/349-cpi-2018\#

40 OANDA. Currency converter: foreign exchange rates, 2018. Available: www.oanda.com

41 Niwa T, Watanabe T, Goto T, et al. Daily review of antimicrobial use facilitates the early optimization of antimicrobial therapy and improves clinical outcomes of patients with bloodstream infections. Biol Pharm Bull 2016;39:721-7.

42 Dik J-WH, Hendrix R, Friedrich AW, et al. Cost-minimization model of a multidisciplinary antibiotic stewardship team based on a successfu implementation on a urology ward of an academic Hospital. PLoS One 2015;10:e0126106.

43 Miyake L, Salgado B, Gomes-da-silva MM. A cost-effectiveness analysis of two different antimicrobial stewardship programs. Brazilian J Infect Dis 2016;20:255-61.

44 Coulter S, Roberts JA, Hajkowicz K, et al. The use of bloodstream infection mortality to measure the impact of antimicrobial stewardship interventions: assessing the evidence. Infect Dis Rep 2017:9:8-12.

45 Holmes AH, Moore LSP, Sundsfjord A, Steinbakk M, et al. Understanding the mechanisms and drivers of antimicrobia resistance. The Lancet 2016;387:176-87.

46 Coulter SA. An economic evaluation of antimicrobial stewardship programs in metropolitan Australian hospitals. dissertation. Queensland, Australia: Queensland University of Technology, 2018. https://eprints.qut.edu.au/116505/

47 Robineau O, Robert J, Rabaud C, Bedos P, et al. Management and outcome of bloodstream infections: a prospective survey in 121 French hospitals (SPA-BACT survey). Infect Drug Resist 2018;11:1359-68. 